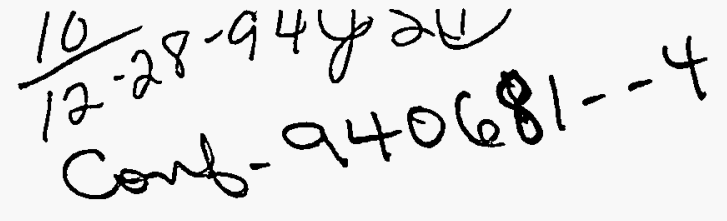

SLAC-PUB-6660 September 1994

\section{MANIFOLD DAMPING OF THE NLC DETUNED ACCELERATING STRUCTURE*}

N. Kroll ${ }^{\dagger}$, K. Thompson, K. Bane, R. Gluckstern ${ }^{\ddagger}$, K. Ko, R. Miller, and R. Ruth Stanford Linear Accelerator Center, Stanford University, Stanford, CA 94309 USA $\dagger$ Physics Department, University of California, San Diego, La Jolla, CA 92093 $¥$ Physics Department, University of Maryland, College Park, MA 20742

\section{Abstract}

In order to mitigate the reappearance of the HOM wakefield of a detuned accelerator structure and relax tolerance requirements, we propose to provide low level damping by coupling all cavities to several identical and symmetrically located waveguides (manifolds) which run parallel to each accelerator structure and are terminated at cach end by matched loads. The waveguides are designed such that all modes which couple to the acceleration mode are non-propagating at the acceleration mode frequency. Hence the coupling irises can be designed to provide large coupling to higher frequency modes without damping the acceleration mode. Because the higher order modes are detuned, they are localized and have a broad spectrum of phase velocities of both signs. They are therefore capable of coupling effectively to all propagating modes in the waveguides. Methods of analyzing and results obtained for the very complex system of modes in the accelerating structure and manifolds are presented.

Presented at the 6th Workshop on Advanced Accelerator Concepts Lake Geneva, WI, June 12-18, 1994

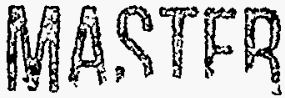

"Work supported by Department of Energy contracts DE-AC03-76SF00515 and DE-FG03-93ER40759.

$$
\text { de }
$$

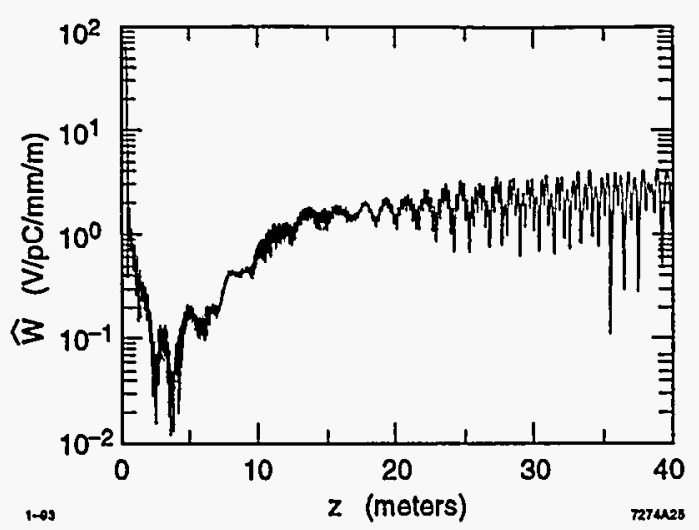

Figure 1: The wakefield envelope function computed from the double band equivalent circuit of Ref. 3. The detuning distribution is gaussian (for parameters see Ref. 1) with a $Q$ of 6500 assumed.

\section{INTRODUCTION}

The acceleration cavities [1] for the NLC test accelerator (NLCTA) are disk-loaded structures composed of 204 cells plus two couplers, 1.8 meters in length, and driven at $11.424 \mathrm{GHz}$. In order to suppress the transverse wakefield the structures are detuned. That is, the cell radius, iris radius, and iris thickness [2] are varied in a smooth (gaussian) way, with the variation of these parameters coupled so as to maintain a uniform cell-to-cell phase advance of 120 degrees for the monopole mode when driven at $11.424 \mathrm{GHz}$. As a result of this variation the widths of the higher order mode (HOM) bands are increased, and the cell-to-cell amplitude variation of the individual modes in a band is drastically altered. As a consequence an off-center charge bunch moving at the velocity of light excites many modes per band rather than the single (synchronous) mode characteristic of a strictly periodic structure, and the average deflecting force experienced by a charge at fixed trailing distance $s$ falls off sharply in $s$ at a rate proportional to the frequency spread of the band. The predicted behavior of this wakefield over distances of interest is illustrated in Fig. 1. It is based on the two-band equivalent circuit model [3] and includes an estimate of the effect of wall losses. While the wakefield does indeed exhibit an initial rapid fall off, it is seen to reappear after a few meters [4]. This effect is associated with the fact that the mode distribution within a band is discrete rather than continuous, and as the elapsed time begins to exceed the inverse mode separation the suppressing effect of a smooth gaussian distribution is lost. As discussed in Ref. 1 , this reappearance can be postponed by interleaving the detuning among four such structures so as to lead to a factor four increase in the effective mode spectrum density. However, submicron tolerances must be imposed in order to actually achieve this extended suppression.

We report in this paper the current status of our investigation of the possibility of suppressing the wake reappearance by providing relatively weak damping via the vacuum manifolds. Our proposed structure is illustrated in Fig.2. The four vacuum manifolds running the length of the structure also function as multimode waveguides which serve to drain power from

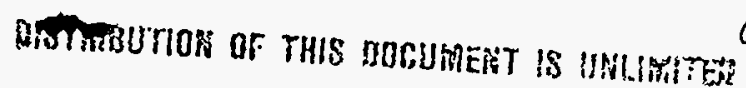


t.a.

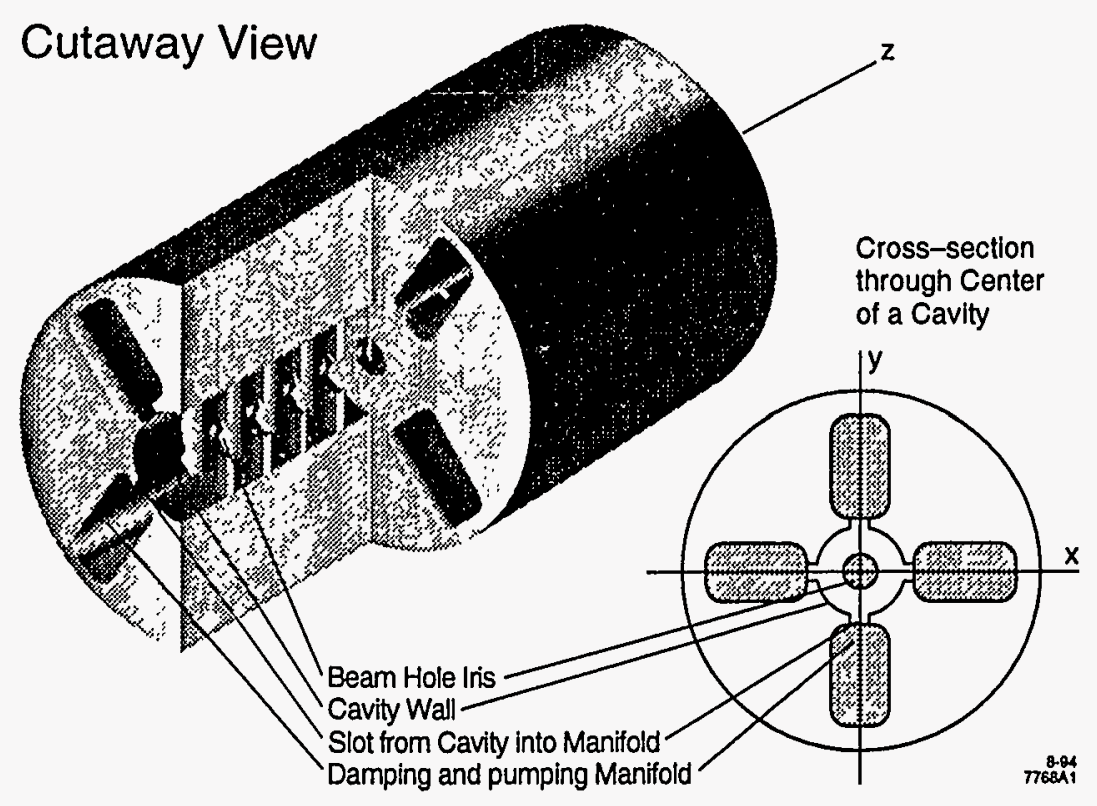

Figure 2: The damped detuned structure.

the HOM's through the large coupling slots located in each cell, except for a few at each end of the manifolds. This omission of coupling at the ends is possible because analysis has shown that the contribution of these cells to the wakefield is negligible, and it enables us to insert absorbing material at each end, intended to serve as matched loads, in a convenient way.

As will be explained in the next section, the waveguides are designed so that only a single mode propagates at $11.424 \mathrm{GHz}$, and that mode is totally decoupled from the acceleration mode by its symmetry. As a consequence the size of the coupling slots is limited only by their effect on the shunt impedance of that mode. We have set a limit of 5\% degradation as a working figure, which limits the space occupied by the slots to approximately one third of the cell circumference. The effectiveness of the manifolds as dampers for the HOM's is connected to the detuning in an essential way. The HOM's of the detuned structure are localized standing waves with spatial variation of effective wavelength which varies smoothly and extensively along the length of the mode. This is precisely the feature which allows many modes in a band to be excited by a velocity of light particle. The excitation pattern of the mode as seen by the waveguide is such that it can drive a manifold wave at a broad range of phase velocities. The coupling of a cavity mode to the manifold is strongest when this range of phase velocities overlaps the phase velocity of one or more manifold modes at the frequency of the cavity mode. As will be seen below, the manifolds have been designed so that this is the case, and the calculations to be described indicate that $Q$ values in the range 500 to 1000 can be achieved for most and perhaps all of the modes which contribute significantly to the wakefield.

\section{MANIFOLD AND DETUNED DIPOLE MODES}

From Fig. 2 we see that the damping waveguides are approximately rectangular in cross section, and consequently their modes can be designated by rectangular waveguide notation. Also the mode order is similar except that the degeneracy between $\mathrm{TM}_{m n}$ and $\mathrm{TE}_{m n}$ modes is broken with the TM modes lying lower. The cutoff frequencies of the set of potentially relevant modes are listed in Table 1 .

\begin{tabular}{ll} 
Table 1: Manifold mode cutoff frequencies \\
\hline \hline $\mathrm{TE}_{10}$ & $6.042 \mathrm{GHz}$ \\
$\mathrm{TE}_{01}$ & $11.507 \mathrm{GHz}$ \\
$\mathrm{TE}_{20}$ & $11.926 \mathrm{GHz}$ \\
$\mathrm{TM}_{11}$ & $12.645 \mathrm{GHz}$ \\
$\mathrm{TE}_{11}$ & $13.464 \mathrm{GHz}$ \\
$\mathrm{TM}_{21}$ & $16.076 \mathrm{GHz}$ \\
\hline \hline
\end{tabular}

We see that the only mode which is propagating at $11.424 \mathrm{GHz}$ is the $\mathrm{TE}_{10}$. Referring to Fig. 2, we see that the structure is symmetric with respect to reflection in the $x z$ and $y z$ planes. The accelerating mode and the $\mathrm{TE}_{10}$ (in any of the manifolds) have opposite behavior under one or the other of these refiections. Hence there can be no coupling between this manifold mode and the accelerating mode, and therefore the manifolds do not damp the accelerating mode. MAFIA plots of the accelerating mode show that the penetration of the fields into the manifolds is weak and confined to the region near the slots. The frequencies of these modes as a function of phase advance per period are shown in Fig. 3.

We now turn our attention to the dipole modes. Figure 3 also exhibits dispersion curves of the first two dipole modes for the first, middle, and last cells of the 206 cells of the detuned structure, cells 1,103 , and 206. Thus it is apparent that the dispersion curve for each cell crosses the dispersion curve of several manifold modes. To qualitatively explain the damping process we turn our attention to the damping and excitation of a single cavity mode. This mode is represented in Fig. 3 by a horizontal line corresponding to its frequency. This particular mode is localized because its mode line extends over the full phase range without intersecting the first or last cell, the excited cells being those whose dispersion curves intersect the mode line. The value of the phase advance at which the dispersion curve of a particular cell crosses the mode line corresponds roughly to the cellto-cell amplitude variation in its vicinity. Thus because the light line crosses the cavity mode line near a particular cell, we expect that the principal excitation of this mode is due to the interaction of the drive bunch with cells in that vicinity. Similarly, there is a cell neighborhood associated with each manifold mode crossing, where effective coupling to the crossing mode may occur. The lower dipole band also has mode lines which terminate at the first or last cell before reaching the $180^{\circ}$ phase advance limit. These modes extend to the first or last cell but terminate in the interior of the structure. It is easy to see that every cavity mode line of the lower band which crosses the light line must also cross the dispersion curve of every propagating manifold mode. The upper dipole mode has cavity mode lines which terminate on the first and last cell. These cavity modes are not localized 


\section{DISCLAIMER}

Portions of this document may be illegible in electronic image products. Images are produced from the best available original document. 


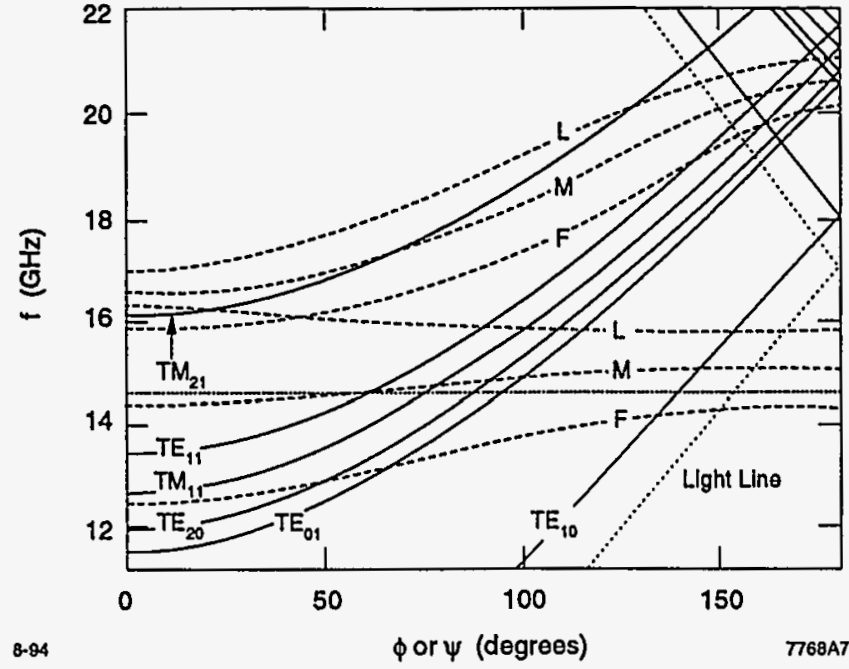

Figure 3: Dispersion curves for the manifold modes designated in Table I overlaying dispersion curves for the first two dipole modes of the first $(F)$, middle $(M)$, and last $(L)$ cells. A cavity mode line (horizontal dotted line) is also shown.

and have a limited phase advance range. There are fewer manifold mode crossings, but there do not appear to be any cases for which a cavity mode which crosses the light line has less than two manifold mode crossings. It should be mentioned that the upper dipole modes in the light line region are largely TE in character (Ref. 3 ) and hence contribute only weakly to the wakefield.

\section{EQUTVALENT CIRCUIT MODEL}

The quantitative results that we report in this paper have all been obtained with the following simplified model. Following [3] we start with a single circuit chain in which each circuit corresponds to a single cell mode and the cells are coupled magnetically. The coupled equations for the cell excitation amplitudes $a_{m}(m=1, \ldots, N)$ are

$$
\left(\frac{1}{f_{m}^{2}}-\frac{1}{f^{2}}\right) a_{m}+\frac{k_{m+\frac{1}{2}}}{2} a_{m+1}+\frac{k_{m-\frac{1}{2}}}{2} a_{m-1}=0 .
$$

Here $f_{m}$ is the resonant frequency of cell $m, f$ is the coupled mode frequency, and the $k$ 's represent the equivalent coupling coefficient between adjacent cells. For the strictly periodic case with coupling coefficients $K$ and cell frequency $F$ one gets the usual solution with constant phase advance per cell $\psi$ and frequency $f$ related to $\psi$ by

$$
1 / f^{2}=1 / F^{2}+K \cos \psi
$$

We describe the manifold modes as transmission lines and use standard transmission line equations with a series reactance per unit length $X$ and shunt susceptence per unit length $B$. For TM modes we take the usual $2 \pi f C$ for $B$, but $2 \pi f L\left[1-\left(f_{c} / f\right)^{2}\right]$ for $X$. Here $f_{c}$ is the cutoff frequency, $c$ is the velocity of light, and $L C=1 / c^{2}$. The propagation constant $k_{8}=\sqrt{X B}$ then takes the correct waveguide form and the characteristic impedence $Z=\sqrt{X / B}=Z_{0}\left(k_{8} / k\right)$ has the correct TM mode frequency dependence. $Z_{0}$, which is the infinite frequency limit of $Z$, will be amalgamated with the cell-waveguide coupling parameter. For the TE case it is $B$ rather than $X$ which acquires the $\left[1-\left(f_{c} / f\right)^{2}\right]$ factor, and $Z=Z_{0}\left(k / k_{g}\right)$ has the correct TE form.

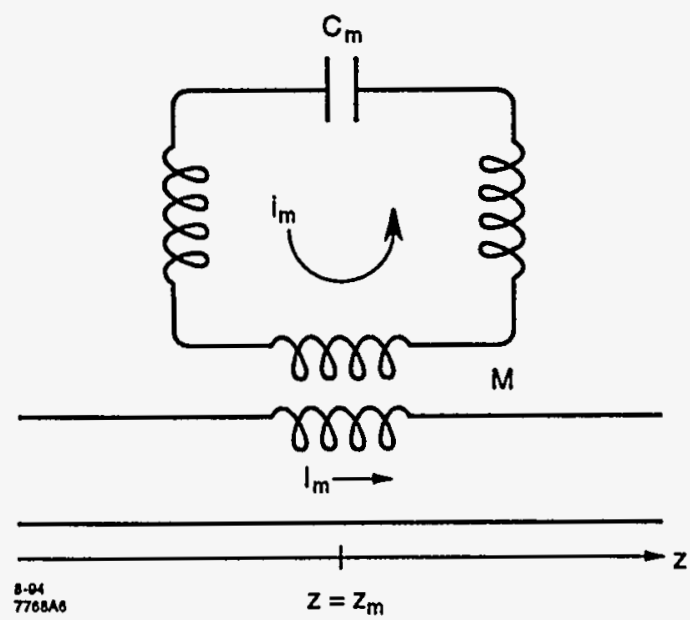

Figure 4: Equivalent circuit representation of the coupling of a single cell to a manifold mode.

The $\mathrm{TE}_{m n}$ manifold modes $(n>0)$ and the TM manifold modes couple to the cell modes via their transverse magnetic field. Since these are generated by axial currents we model the coupling via a mutual inductance $M$ between an inductance $L_{c}$ in the cell and an inductance $L_{t}$ in the transmission line as shown in Fig. 4. We first consider the case in which only one cell, the $m$ th, is coupled to the line. It is easy to show that the current induced in the transmission line by a current $i_{m}$ in the cell may be written

$$
I_{i}(z)=I_{m} \exp \left(-j k_{g}\left|z_{m}-z\right|\right),
$$

where

$$
I_{m}=-\frac{j w M_{m}}{\left(2 Z+j w L_{\ell}\right)} i_{m}
$$

and $z_{m}$ represents the location of the coupling slot on the transmission line. If we couple other cells to the line, the lumped line inductances $L_{t}$ associated with this coupling modifies the transmission characteristics of the line, and Eq. 3 would have to be modified even if all of the other $i_{n}$ are zero. For the calculations reported in this section these inductances 


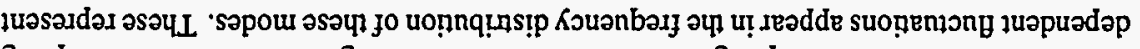

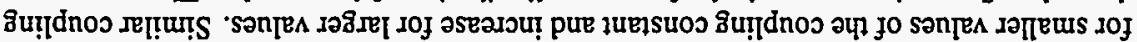

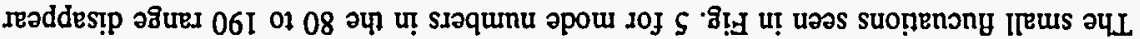

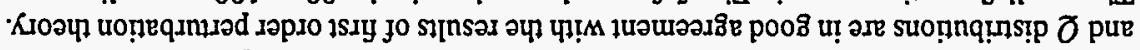

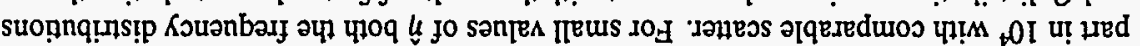

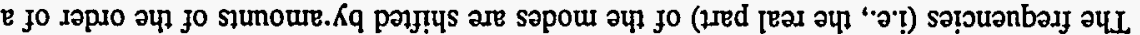

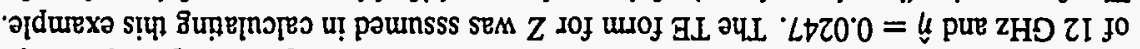

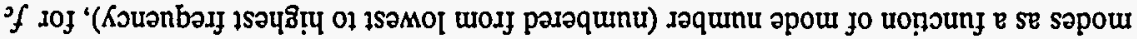

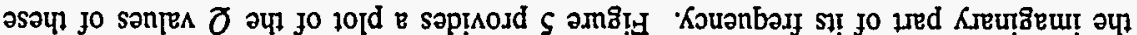

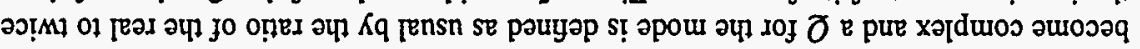

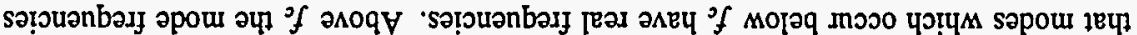

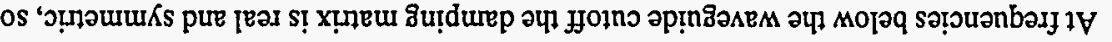

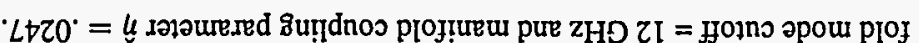

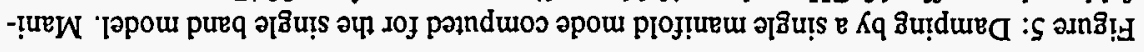

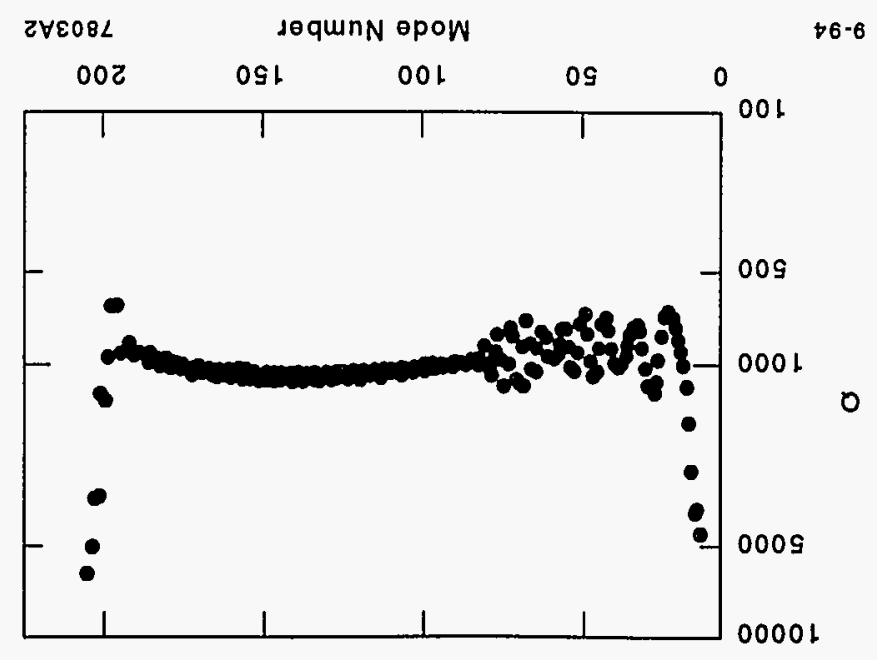

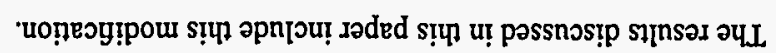

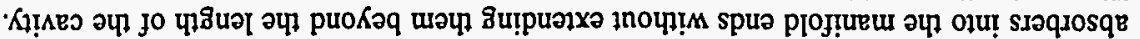

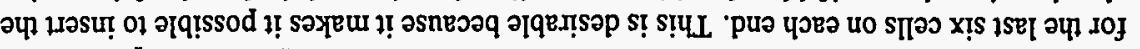

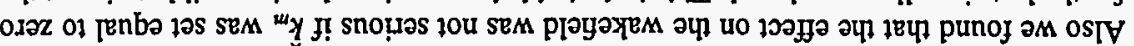

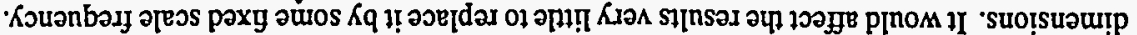

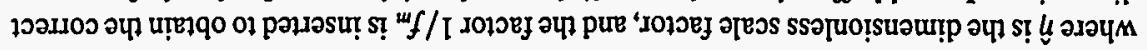

$$
\text { - }{ }^{m} f / u={ }^{u} y
$$

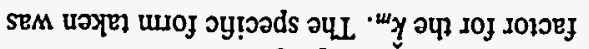

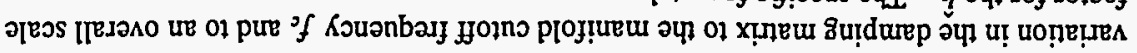

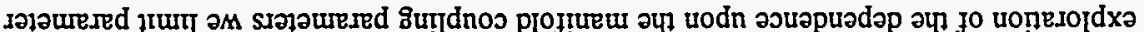

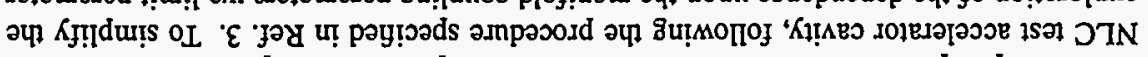

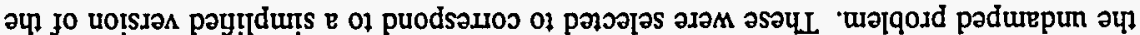

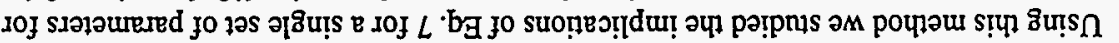

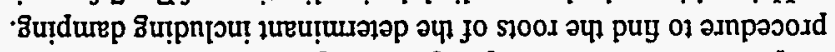

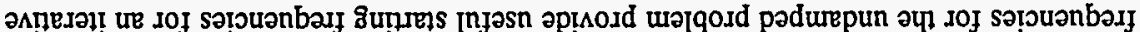

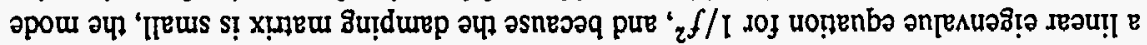

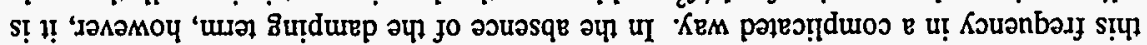

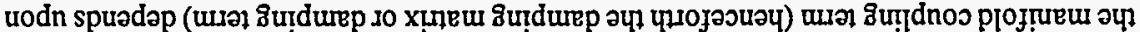

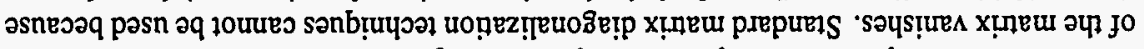

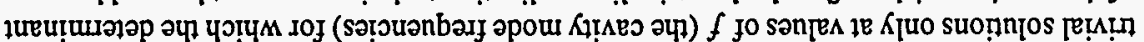

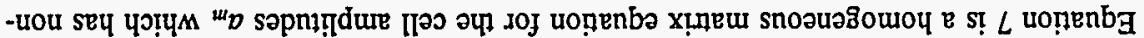

\section{THCOW HHI GO SNOILVDITddV}

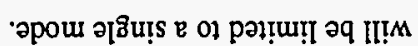

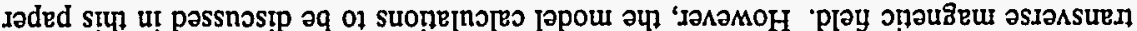

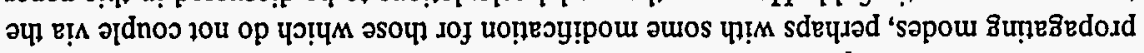

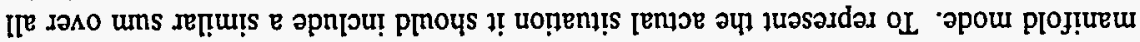

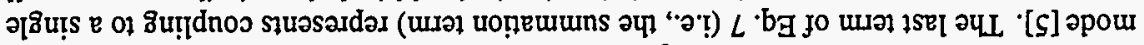

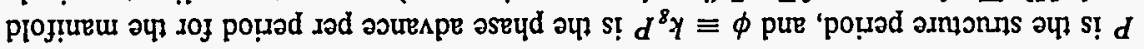

$$
\cdot\left({ }^{0} Z d \tau\right) / \nu^{w} \supset{ }^{\mu w} W \mu \tau \equiv{ }^{w} y
$$

ว.әчM

$(L)$

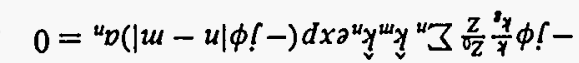

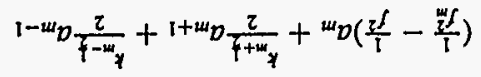

suoṇenbo pođđnos jo ras

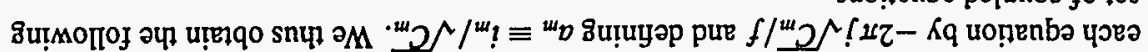

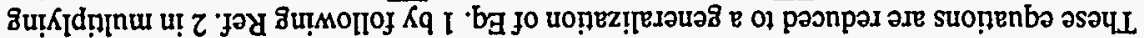

(9)

$$
\begin{aligned}
& \text { - } 0=\left({ }^{\mu} z\right)^{\prime} I^{\mu} W m !+
\end{aligned}
$$

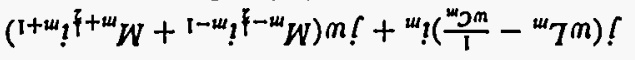

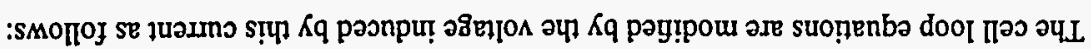

$$
\text { - }\left(\left|z-{ }^{u} z\right|^{8} y !-\right) \mathrm{dx}{ }^{u} ?^{u} W \stackrel{u}{\zeta} \frac{z z}{m !}-=(z)^{\prime} I
$$

juaunn out! uo!sstursuren

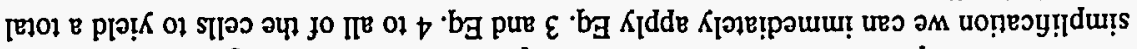

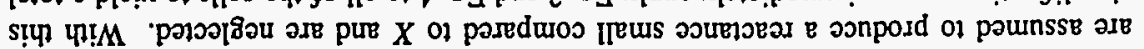


the main effect of the departure from perturbation theory.We note that $Q$ 's of the order of 1000 correspond to a resonance width, $f / Q$, twice as large as the mode separation at the peak of the mode spectrum. Thus substantial distortion of the mode patterns is expected and because the group of cells within the mode which are well coupled to the manifold mode is localized, a tendency of the modes to split into more weakly and more strongly damped groups is perhaps not surprising. If this interpretation is correct, we should see smoother results when more manifold modes are taken into account. We have also varied $f_{c}$ from zero to $12 \mathrm{GHz}$. We find that those modes whose mode lines cut the light line are damped about as well as those shown in Fig. 5, but there are a substantial number of very weakly damped modes at the low frequency end associated with the fact that their mode lines do not cut the manifold mode dispersion curve. These results support the physical interpretation of the damping process given in section 2 .

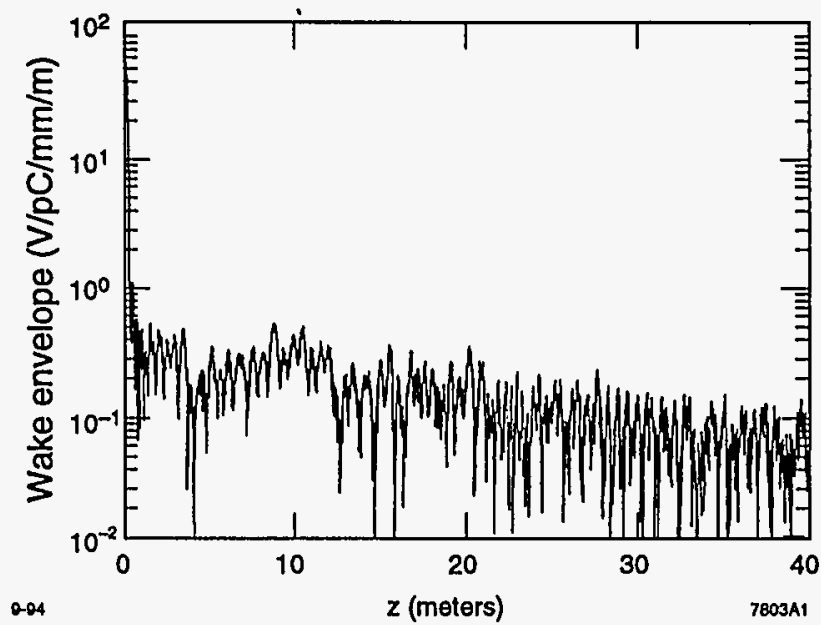

Figure 6: Wakefield for the parameters used for Fig. 5. The effect of copper losses have been included by combining the $Q$ values of Fig. 5 with an assumed copper loss value of 6500 .

Figure 6 shows the wakefield computed for the Fig. 5 parameters using the $Q$ values and perturbed frequencies with the kick factors of the undamped modes. It would be straightforward to obtain the perturbed amplitude distribution functions and to calculate the wakefield from them, but this calculation has not yet been carried out. Comparing to Fig. 1 , we see that the reappearance at large distances has been completely suppressed [6]. The deep minimum shown in Fig. 1 has also disappeared. The latter resembles an effect which had been observed when the tolerance requirements for the undamped structure were studied and is believed to be due to the scatter in the perturbed frequencies. The deep minimum is not needed for beam stability, however, and the wakefield of Fig. 6 is considered to be satisfactory.

\section{MANIFOLD COUPLING PARAMETERS}

We discuss here a method we have used to relate the manifold coupling parameter $\hat{\eta}$ to a physical structure. We have used MAFIA to study the spectrum of single cells coupled to the manifolds as a function of phase advance per period. Since this corresponds to the properties of a periodic structure we specialize Eq. 7 to this case. Thus we set $f_{m}=F$, $k_{m}=K$, and $a_{m}=A \exp (j \psi m)$. The sum may then be carried out and in the TE case the resultant equation takes the form

$$
\left(\frac{1}{F^{2}}-\frac{1}{f^{2}}+K \cos \psi\right)(\cos \psi-\cos \phi)=\hat{\eta}^{2} \phi \frac{\sin \phi}{F^{2}} .
$$

In the absence of manifold coupling the RHS of Eq. 10 is zero, and a plot of the solution $f(\psi)$ would show two curves, the cavity dispersion curve associated with the vanishing of the first factor on the LHS and the waveguide dispersion curve with that of the second. Since the coupling term will be small, it perturbs this pair of curves very little except near the point where both factors vanish. Because the RHS of the equation is not zero, the two curves cannot cross, and thus one gets typical avoided crossing behavior. The detailed form of the curves in the vicinity of the avoided crossing is very sensitive to the value of $\hat{\eta}$.

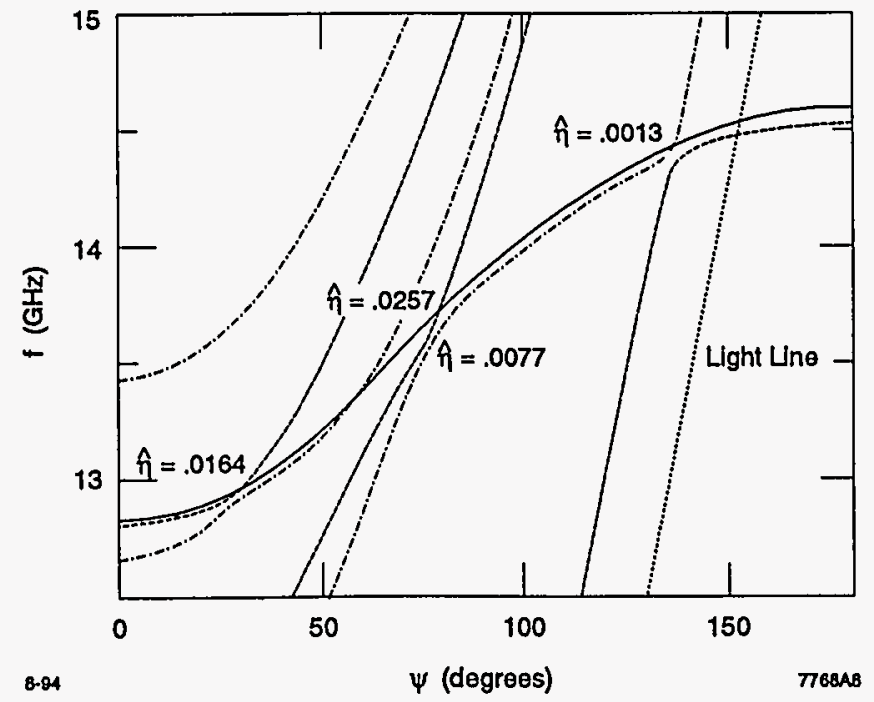

Figure 7: MAFIA computed mode frequencies for cell 10 of the detuned structure coupled to four manifolds with a set of phase advances per period specified. The frequency range shown restricts the figure to the lower dipole mode and to the avoided crossing manifold modes. The shunt impedence degradation of the accelerating mode is 2\%; coupling constants $\hat{\eta}$ computed for each avoided crossing are shown on the figure.

Figure 7 is obtained from a series of MAFIA runs for a single structure but for varying specifications of phase advance. The frequency range is limited in the plot so that only 
a single cell mode is shown. A number of avoided crossings are evident associated with different manifold modes. We assume that the crossings can be treated as though wel separated from one another, and that the behavior in the immediate vicinity of each crossing can be fit with Eq. 10. The fitting is done by choosing two points at each of two $\psi$ values, near to but on either side of the point of closest approach. This provides four input point pairs which are then used to determine all four parameters in Eq. 10. For our curren preliminary assessment we have applied Eq. 10 to all of the crossings, regardless of the type of the manifold mode. The results of this procedure are shown on Fig. 7. We have carried out such an analysis for four different cell designs intended to approximate the curren detuned structure design. The beam iris design corresponds precisely to particular cells. The coupling slots are taken to be of uniform width and thickness and with length equal to the distance between beam irises. Because of the coupling slots the cell radius has to be reduced so as to maintain the proper phase advance for the accelerating mode. The values found for the effective coupling vary with the cell, but the $\hat{\eta}=0.0247$ used in Figs. 5 and 6 is quite easy to obtain in the proposed geometry. Furthermore the average degradation of the shunt impedence for the four cells is $2.3 \%$, consistent with the working limit that we set.

\section{FUTURE PLANS AND CONCLUDING REMARKS}

While the theory presented above is quite crude, it does allow us to hope that an effective damped detuned structure can be designed along the lines described in this paper. Some obvious theoretical improvements are planned or in progress. These include the improved manifold theory [5] and coupling all of the relevant modes at once, taking account of the differences in coupling behavior of the various types of cavity modes. The analysis should be repeated for the double band model taking account of the difference in the way the TE and TM components of the cell modes couple to the manifold modes.

An experimental program involving a full scale version of a damped detuned structure is being planned. The presence of the manifolds enhances cold test opportunities. Because of the localization of the important detuned modes within the structure it has not been possible to observe them directly with cold test procedures. The manifolds provide access to all of them, and it should be possible to demonstrate HOM damping via RF transmission from one manifold to another through the cavity cells. Direct observation of the wakefield as in [4] is of course also planned. Observation of the frequency spectrum of the beam induced radiation out of the manifold will also provide valuable beam diagnostic information.

We thank the other members of the NLC structures group for useful dicussions and comments.

\section{REFERENCES}

1. K.A. Thompson, et.al., SLAC-PUB-6032, November 1993, and references therein. To appear in Particle Accelerators.

2. We will be concerned in this paper primarily with the first two dipole bands. These are effectively detuned without thickness variation. Thickness variation is introduced to detune the TEM $\mathrm{T}_{m n 1}$ modes as well.

3. Karl L.F. Bane and Robert L. Gluckstern, Part. Accel. 42, 123 (1993); SLAC-PUB-5783. 4. The wakefield of an accelerator cavity built as specified in [1] has recently been measured and found to be in reasonable agreement with Fig. 1, although the reappearance effect is somewhat less than expected. See C. Adolphsen, et.al., submitted to Phys.Rev.Lett; see also SLAC-PUB-6629.

5. The series inductance which we have omitted can be included in a straightforward way by treating the waveguide as a periodic structure formed by sections of transmission line of length $P$ in series with the inductances. This leads to small modifications in the expressions for $\phi$ and $Z$ that can easily be included in the above treatment. A small gap in frequency, within which the mode does not propagate, appears between the forward and backward wave branch of each manifold mode. This gap is seen in the MAFIA simulations and can be used to determine the parameter associated with the series inductance.

6. Because the frequency fluctuations increase with increasing coupling strength, the magnitude of the wakefield at short distances may increase to unacceptable values for unnecessarily large values of $\hat{\eta}$. This effect rather than shunt impedence degradation of the accelerating mode may limit the size of the coupling slots.

\section{DISCLAIMER}

This report was prepared as an account of work sponsored by an agency of the United States Government. Neither the United States Government nor any agency thereof, nor any of their employees, makes any warranty, express or implied, or assumes any legal liability or responsibility for the accuracy, completeness, or usefulness of any information, apparatus, product, or bility for the accuracy, completeness, or usefulness of any information, apparatus, product, or ence herein to any specific commercial product, process, or service by trade name, trademark, manufacturer, or otherwise does not necessarily constitute or imply its endorsement, recommendation, or favoring by the United States Government or any agency thereof. The views and opinions of authors expressed herein do not necessarily state or reflect those of the United States Government or any agency thereof. 